\title{
Metallophthalocyanine-nanofibre-based electrodes for electrochemical sensing of biomolecules
}

\author{
K PRIYA MADHURI ${ }^{1,2}$ and NEENA S JOHN ${ }^{1, *}$ \\ ${ }^{1}$ Centre for Nano and Soft Matter Sciences, Jalahalli, Bangalore 560013 , India \\ ${ }^{2}$ Department of Chemistry, Mangalore University, Mangalagangotri 574 199, India \\ *Author for correspondence (jsneena@cens.res.in)
}

MS received 20 March 2018; accepted 9 July 2018; published online 27 August 2018

\begin{abstract}
Metal phthalocyanines, possessing rich redox chemistry due to the presence of the central metal cation and pyrrolic nitrogen atoms of the macrocycle, are explored as electrochemical sensors. Nickel phthalocyanine nanofibres (NiPc $\mathrm{NF}$ ) prepared by a simple chemical route are coated on a pencil graphite rod and the electrocatalytic performance of $\mathrm{NiPc} N F$ electrode is investigated for quantitative detection of ascorbic acid (AA) in $0.2 \mathrm{M}$ phosphate buffer solution. The performance of NiPc NFs is shown to be superior to that of commercial NiPc and is attributed to the high electrochemically active surface area available for fibres. The electrode exhibits linearity for the detection over a wide concentration range of AA from 5.5 $\mu \mathrm{M}$ to $5.2 \mathrm{mM}$. The detection limit for AA sensing with NiPc-NF-modified electrode is $1.5 \mu \mathrm{M}$. The higher performance of $\mathrm{NiPc}$ fibres due to its nanostructure morphology may be utilized for the quantitative detection of other biomolecules.
\end{abstract}

Keywords. Nickel phthalocyanine; nanofibres; ascorbic acid; electrochemical oxidation.

\section{Introduction}

Metal phthalocyanines (MPcs) are highly delocalized aromatic systems possessing metal ions at the centre of the macrocycle. They are structural analogues of porphyrins and exhibit unique physicochemical properties, which depend on their molecular packing [1]. Apart from being thermally and chemically stable, they also possess rich redox chemistry, which enables them to be useful candidates for electrochemical applications including electrocatalysis, supercapacitance, fuel cells, etc. [2,6-9]. MPcs containing transition metals are mostly planar and exhibit intense absorption ranging from ultraviolet to near-infrared region and are thus useful in sensitization of large-bandgap semiconductors in solar photovoltaics [5]. Since the native MPcs are not electrically conductive, the applications often include the usage of conductive carbon materials or polymers to enhance their properties. Substitution of MPcs with active functional moieties also enhances certain characteristics of MPcs, like better solubility, conductivity, electron-hole donor properties, etc. [3,6-9]. Applications involving native unsubstituted MPcs are rare.

Commercially obtained unsubstituted MPcs are large crystallites that are insoluble in most common solvents and cannot be easily dispersed. Thus, device fabrication for electrochemical applications becomes challenging due to less viable solution-processable methods. Increasing demand for improved device performance has resulted in the development of tailored nanomaterials over the past few years. Nanomaterials possess significantly superior properties like surface area to volume ratio when compared with their conventional forms, resulting in higher chemical activity. Since MPcs can self-assemble and form supramolecular structures through $\pi-\pi$ stacking, it is easy to fabricate them in an elongated fashion. Researchers have fabricated various morphologies of MPcs through surfactant-assisted synthesis to yield spherical nanostructures [10], solvothermal synthesis for 3D-flowerlike structures [11] and controlled evaporation techniques to obtain nanoribbons and nanorods [12]. Electrochemical deposition and laser ablation methods to achieve needle-like nanostructures are also known $[13,14]$.

This work deals with the study of the role of morphology of MPcs in electrochemical applications. Nickel phthalocyanine (NiPc) nanostructures synthesized by a simple chemical method are easily dispersible in polar solvents for facile preparation of electrodes. In an earlier report from our group, we showed that NiPc nanofibre (NiPc NF)-modified graphite electrode in combination with reduced graphene oxide provides excellent supercapacitive performance [15]. In this work, the use of unsubstituted NiPc NF as an electrochemical sensor is demonstrated for the detection of ascorbic acid (AA) using cyclic voltammetry $(\mathrm{CV})$ and differential pulse voltammetry (DPV) measurements and its performance is compared to that of commercially obtained NiPc powder.

\section{Experimental}

\subsection{Chemicals}

Nickel chloride hexahydrate $\left(\mathrm{NiCl}_{2} \cdot 6 \mathrm{H}_{2} \mathrm{O}\right)$ (Merck, 97\%), phthalonitrile $\left(\mathrm{C}_{6} \mathrm{H}_{4}(\mathrm{CN})_{2}\right)$ (TCI, >99\%), ammonium 
heptamolybdate $\left(\left(\mathrm{NH}_{4}\right)_{6} \mathrm{Mo}_{7} \mathrm{O}_{24}\right)($ Merck, 83\%), ethylene gylcol $\left(\mathrm{C}_{2} \mathrm{H}_{6} \mathrm{O}_{2}\right)$ (Alfa Aesar, >99\%), L-AA $\left(\mathrm{C}_{6} \mathrm{H}_{8} \mathrm{O}_{6}\right)$ (Sigma-Aldrich, 99\%), monopotassium phosphate $\left(\mathrm{KH}_{2} \mathrm{PO}_{4}\right)$ (Merck, >99\%) and dipotassium phosphate $\left(\mathrm{K}_{2} \mathrm{HPO}_{4}\right)$ (Merck, >98\%).

\subsection{Preparation method}

We followed the synthesis procedure for NiPc NF as reported previously [15]. In brief, nickel chloride and phthalonitrile were taken in 1:4 molar ratio and mixed with a small amount of ammonium heptamolybdate to catalyse the reaction. This mixture was suspended in ethylene glycol by sonication and heated at $100^{\circ} \mathrm{C}$ for $8 \mathrm{~h}$ to obtain a deep-blue-coloured solution. The product was purified by centrifugation and stored as an ethanolic dispersion.

\subsection{Characterization}

Optical properties of NiPc NF in ethanol were studied using a Perkin-Elmer Lambda 750 spectrophotometer. A Raman spectrum for the sample was recorded using a Horiba XploRA PLUS spectrophotometer having a $50 \times$ objective, with a laser source of $532 \mathrm{~nm}$. X-ray diffraction (XRD) was performed using a Rigaku Smartlab X-ray diffractometer having $\mathrm{Cu} \mathrm{K} \alpha$ radiation source. The morphological features of NiPc NF coated on graphite electrode were studied using a TESCAN MIRA3 LM field emission scanning electron microscope (FESEM).

The electrochemical measurements were performed using a $\mathrm{CH}$ Instrument 660E, in a typical three-electrode configuration. A standard $\mathrm{Ag}-\mathrm{AgCl}$ (sat. $\mathrm{KCl}$ ) and platinum wire were used as the reference- and the counter-electrode, respectively, in an aqueous phosphate buffer saline. We use a pencil graphite electrode (PGE) as a working electrode, which is cost-effective and eco-friendly [15]. The obtained graphite rods were initially treated with dilute nitric acid to remove any contaminants and further washed with milli-Q water and dried. Slurry of the active material was prepared by dispersing $\mathrm{NiPc} \mathrm{NF}$ in a polar solvent, dimethyl formamide, and coated onto the tip of PGE. The PGE electrode was assembled in a plastic microtip for mechanical support, through which the copper wire was wound at the other end of the graphite electrode to establish an electrical connection. The dimensions of PGE bearing active material coating were $0.7 \mathrm{~mm}$ diameter and $2.0 \mathrm{~mm}$ height. The electrodes were dried overnight in a vacuum desiccator and weighed before the electrochemical measurements.

\section{Results}

Figure 1 shows the XRD pattern of the as-synthesized NiPc NF material. The ethanolic solution of NiPc was drop casted on silicon substrate and dried. Peaks arising at $2 \theta$ values of $6.82,7.38,12.03,15.88$ and 24.84 belong to diffraction planes

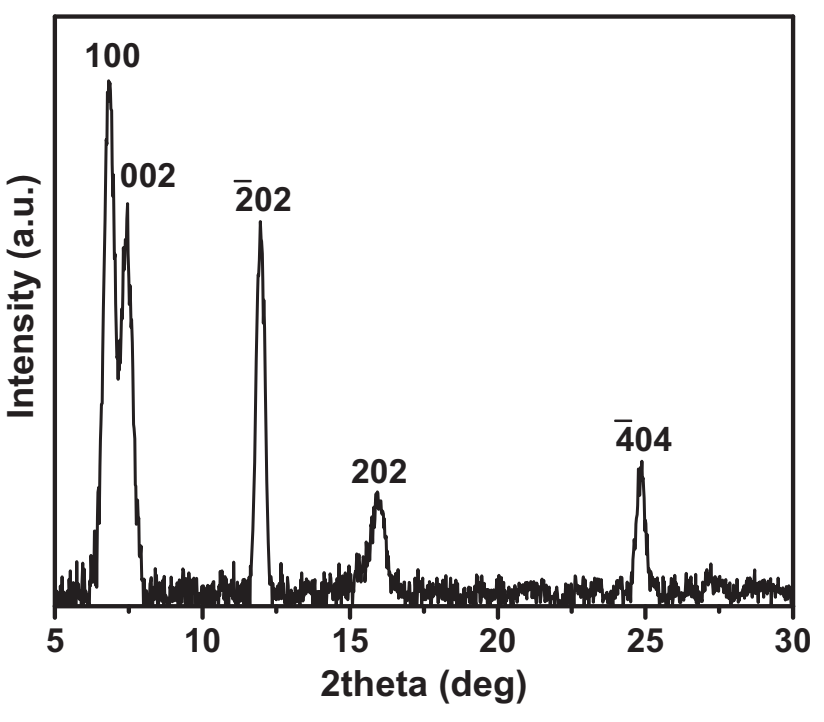

Figure 1. X-ray diffraction pattern of $\mathrm{NiPc} \mathrm{NF}$ on a silicon substrate.

of $100,002, \overline{2} 02,202$ and $\overline{4} 04$, respectively, corresponding to $\beta$ phase of NiPc [17]. Hence, phase-pure NiPc has been obtained during the synthesis of nanostructures whereas the commercial NiPc powder usually consists of both $\alpha$ and $\beta$ phases. The formation of NiPc is also confirmed by obtaining the absorption spectra. The characteristic B bands in the 275$475 \mathrm{~nm}$ region and $\mathrm{Q}$ bands in the $575-875 \mathrm{~nm}$ region, which pertain to metallophthalocyanines, are observed. Raman spectra showed peaks due to phthalocyanine metal cation and macrocycle as described in the earlier study [15]. The most intense band is centred around $1545 \mathrm{~cm}^{-1}$, which arises due to the pyrrole stretching. This band is unique to the individual metal ion accommodating the macrocycle, which causes slight displacements in the $\mathrm{C}-\mathrm{N}-\mathrm{C}$ bonds and thus, Raman shift. Another pyrrole stretching due to $\mathrm{C}-\mathrm{C}$ bonds appears at $1350 \mathrm{~cm}^{-1}$. Other major peaks include benzene and macrocycle deformation in the 500-600 and $750-850 \mathrm{~cm}^{-1}$ regions, respectively.

The morphology and distribution of NiPc NFs coated on the graphite working electrode before and after electrochemical measurements are examined using the FESEM and the images are shown in figure 2. It is seen that the NiPc fibres are coated well on the flaky surface of the graphite electrode with a good packing density of the fibres (figure 2a). The obtained NiPc fibres are on the order of several microns in length and are found to be aggregated to give a mat-like appearance (inset of figure $2 a$ ). Figure $2 b$ shows images of the modified electrode after performing the electrochemical measurements. NiPc fibres are seen to be intact with a very good density, indicating that the electrode has not deteriorated and is stable without any loss of active material even after electrochemical tests (inset of figure $2 b$ ). This proves the high chemical and mechanical stability of NiPc NFs. The elongated morphology of the MPcs is a result of supramolecular self-assembly of the molecules, in which the $\pi$ interactions in 

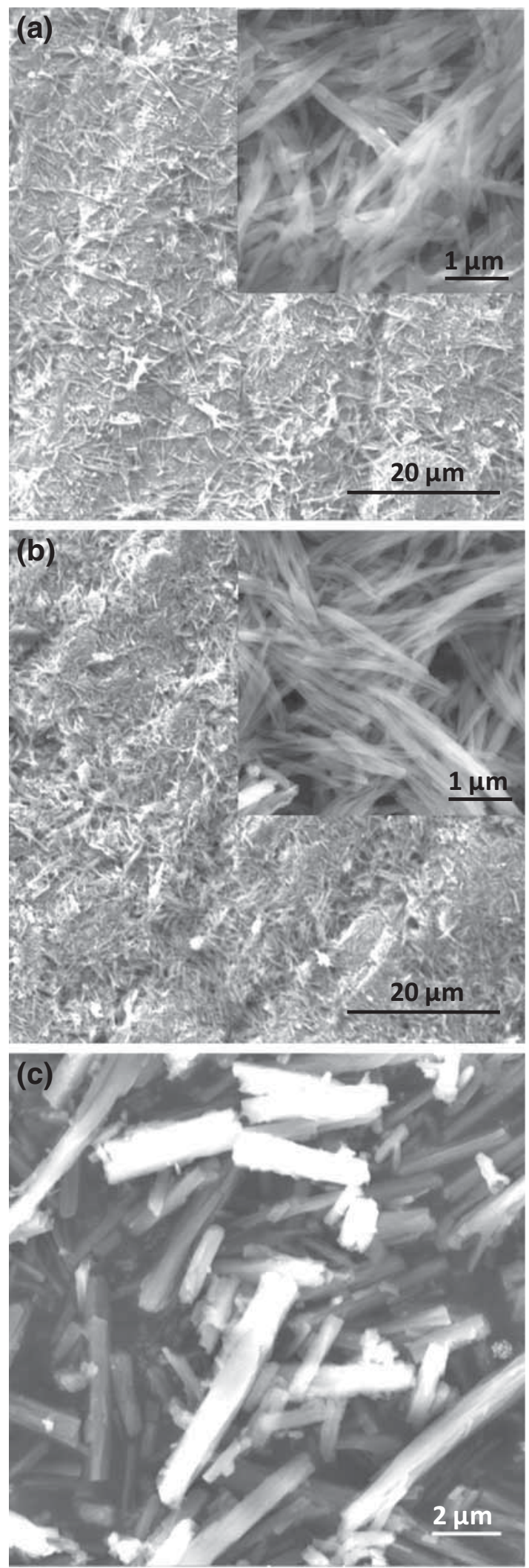

Figure 2. FESEM images of (a) NiPc-NF-coated graphite rod electrode, (b) NiPc-NF-modified graphite electrode after performing the electrochemical experiments (insets show magnified images) and (c) commercially obtained NiPc powder.
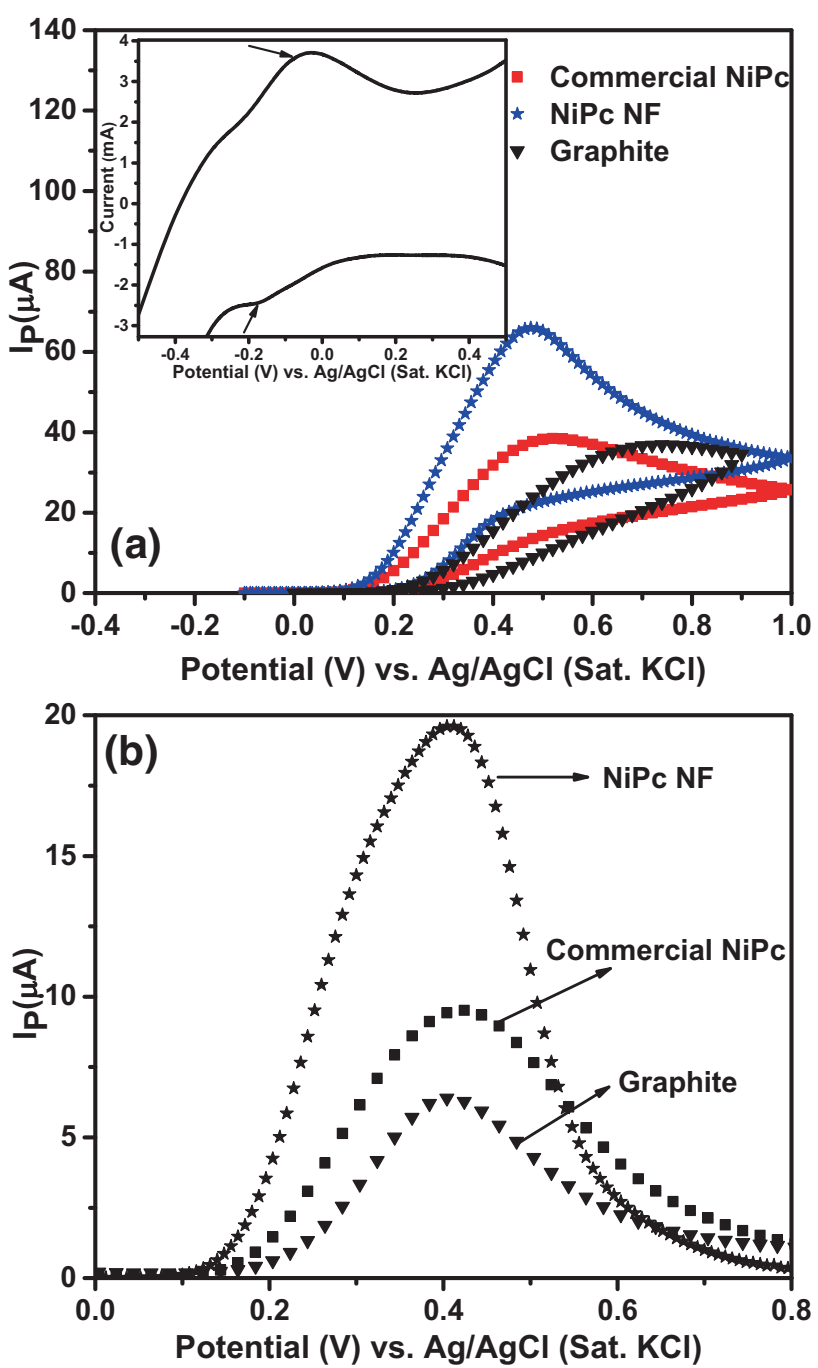

Figure 3. (a) Cyclic voltammograms and (b) differential pulse voltammograms of graphite electrode, commercial NiPc- and NiPcNF-modified PGE electrode in a $0.2 \mathrm{M}$ phosphate buffer solution of $\mathrm{pH} 6$ in the presence of $5 \mathrm{mM}$ ascorbic acid at a scan rate of $0.1 \mathrm{~V} \mathrm{~s}^{-1}$. Inset of (a) is $\mathrm{CV}$ for NiPc-NF-modified electrode in the absence of AA.

these molecules are dominating. However, the control of the width and length of the crystallite becomes important for a particular application. For the synthesized NiPc NF, the width of the fibres is roughly $150 \mathrm{~nm}$ and the length of the fibres is about $5 \mu \mathrm{m}$ or less. The dimensions of the synthesized NiPc $\mathrm{NF}$ are very small compared with the rod-shaped crystallites in commercially obtained NiPc powder having an average width as large as $1 \mu \mathrm{m}$ and length greater than $5 \mu \mathrm{m}$ as shown in figure 2c. More importantly, the NiPc NFs obtained are highly dispersible in most polar organic solvents while the commercial powders suffer from insolubility.

Electrocatalytic behaviour of $\mathrm{NiPc} \mathrm{NF}$ towards the electrochemical oxidation of AA was studied by performing $\mathrm{CV}$ and DPV measurements. The experiment was performed in $0.2 \mathrm{M}$ phosphate buffer solution in the potential 


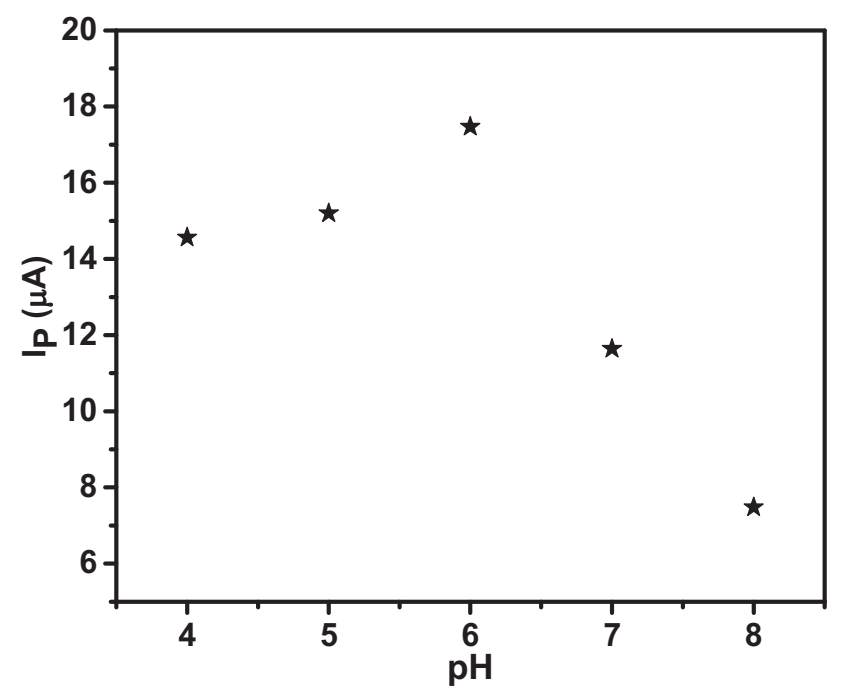

Figure 4. Plot of peak current vs. $\mathrm{pH}$ in the presence of $5 \mathrm{mM}$ ascorbic acid in $0.2 \mathrm{M}$ phosphate buffer solution for NiPc-NFmodified electrode.

range of -0.1 to $1.0 \mathrm{~V} v s$. $\mathrm{Ag}-\mathrm{AgCl}$ (sat. $\mathrm{KCl}$ ) at different $\mathrm{pH}$ conditions for optimization. Figure $3 \mathrm{a}$ shows $\mathrm{CV}$ measurements for all the three electrodes: unmodified PGE, commercial NiPc- and NiPc-NF-modified electrodes, carried out in the presence of $5 \mathrm{mM} \mathrm{AA}$ at $0.1 \mathrm{~V} \mathrm{~s}^{-1}$. The inset given in figure $3 \mathrm{a}$ is the $\mathrm{CV}$ profile for NiPc NF electrode in the absence of AA. It is seen that the redox couple appears around -0.05 and $-0.175 \mathrm{~V}$, which can be attributed to $\mathrm{Ni}$ (II)-Ni (III) redox reaction. In the presence of AA, it is seen that all the three electrodes show a distinct oxidation peak. The absence of reduction peak in the cathodic scan shows that the AA oxidation is irreversible on the surface of variously modified electrodes. The peak positions for AA oxidation slightly differ in all the three electrodes, with the NiPc NF electrode being at slightly lower potential. For unmodified PGE the oxidation peak appears around $0.65 \mathrm{~V}$ and for commercial NiPc and NiPc NF, the oxidation peak appears at $0.43 \mathrm{~V}$. This indicates that the NiPc-modified electrodes have better catalytic activity towards AA oxidation. The overpotential required for driving AA oxidation varies with electrode type. Barros et al [18] report that the carbon ceramic electrode modified by $\mathrm{SiO}_{2}$-graphite-NiPc material catalyses the oxidation of AA at a potential of $0.3 \mathrm{~V}$. Oni et al [19] demonstrated the iron-tetrasulphophthalocyaninemodified carbon paste microelectrode for the detection of AA along with dopamine at $0.35 \mathrm{~V}$. A potential of $0.43 \mathrm{~V}$ in our case is expected for a native NiPc NF electrode. The combination with carbon materials would decrease the overpotential required for AA oxidation to as low as $0.1 \mathrm{~V}$, which was reported by Yang et al [20] using cobalt phthalocyanine and carbon paste electrode.

From figure $3 \mathrm{a}$ it can also be noticed that the intensity of the anodic peak current observed for NiPc NF is higher and well defined when compared with commercial NiPc
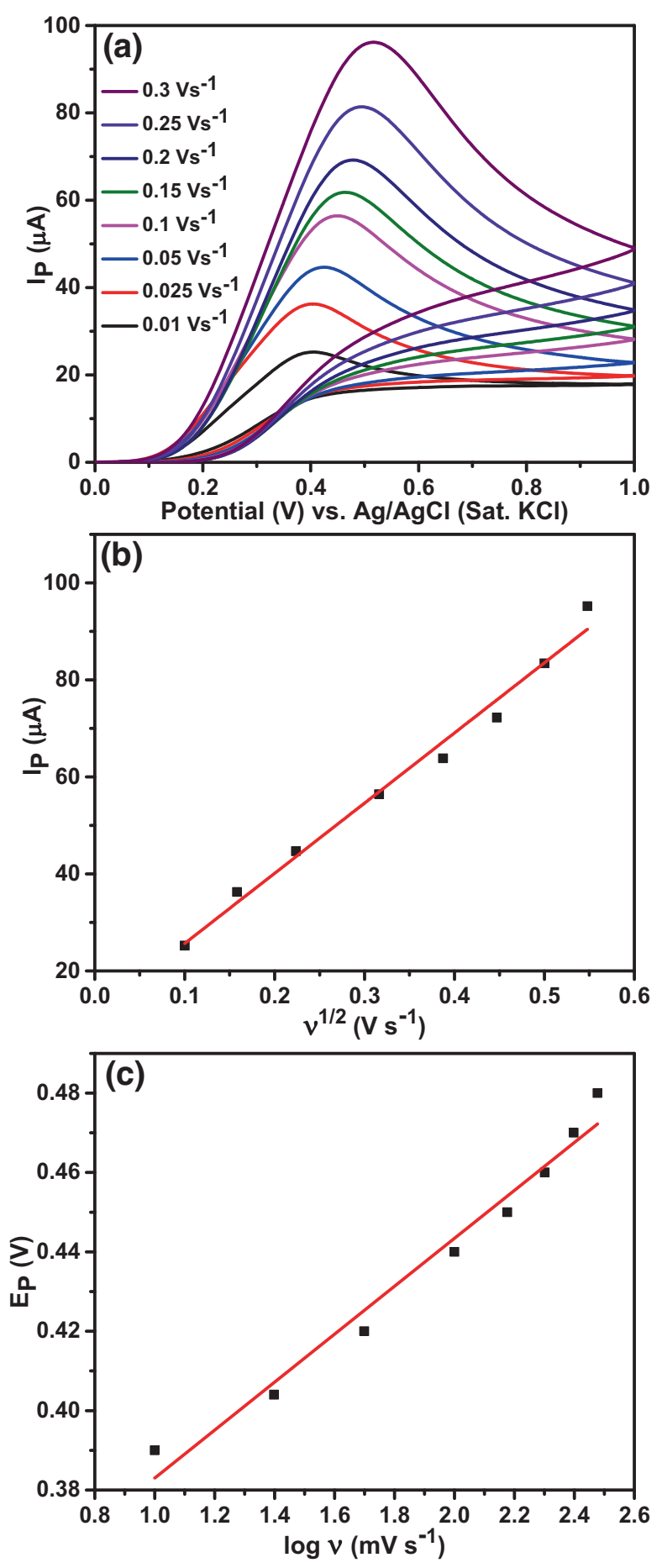

Figure 5. (a) Cyclic voltammograms at various scan rates from 0.01 to $0.3 \mathrm{~V} \mathrm{~s}^{-1}$ of NiPc-NF-modified PGE electrode in a $0.2 \mathrm{M}$ phosphate buffer solution at $\mathrm{pH} 6$ in the presence of $5 \mathrm{mM}$ ascorbic acid, (b) plot of anodic peak current $v$ s. square root of scan rate $v$ and (c) plot of peak potential vs. $\log v$.

powder, indicating that NiPc-NF-modified electrode is highly sensitive to the presence of AA. This was further confirmed from DPV measurements. Figure $3 b$ shows the DPV carried out with bare PGE, commercial NiPc and NiPc NF in the 


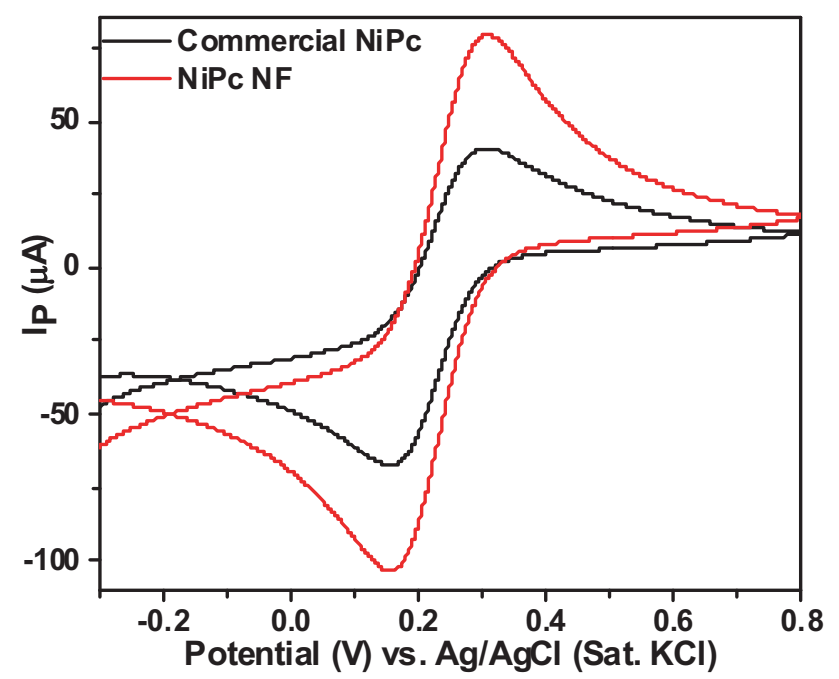

Figure 6. Cyclic voltammograms obtained for $\mathrm{NiPc} \mathrm{NF}$ and commercial NiPc-modified electrodes in the presence of $9 \mathrm{mM}$ $\mathrm{K}_{3} \mathrm{FeCN}_{6}$ in $0.2 \mathrm{M}$ phosphate buffer solution of $\mathrm{pH} 6$ at a scan rate of $0.1 \mathrm{~V} \mathrm{~s}^{-1}$.

presence of $5 \mathrm{mM}$ AA. The oxidation peak arises at a potential of $0.43 \mathrm{~V}$ for both NiPc NF and commercial NiPc-modified electrode, but the peak current is higher in case of NiPc $\mathrm{NF}$ when compared with commercial NiPc. This projects the point that NiPc NF electrodes may be explored for AA sensing.

The oxidation process of AA starts with the diffusion of the analyte from the bulk of the electrolyte solution and adsorption on the surface of electrode. According to literature [21], the most probable mechanism for AA oxidation is the reaction between the central metal ion of the phthalocyanine and the surface-adsorbed AA. Initially, M(II) gets oxidized to M(III), which in turn drives the oxidation of AA by accepting the electrons while regenerating $\mathrm{M}$ (II).

The influence of $\mathrm{pH}$ on the activity of NiPc-NF-modified PGE electrode was investigated to optimize and extract maximum response from the electrode at a specific $\mathrm{pH}$. Figure 4 shows the plot of anodic peak current obtained from DPVs of NiPc NF electrodes at different $\mathrm{pH}$ values. The deviation in peak potentials observed for various $\mathrm{pH}$ is negligible. A maximum anodic current was observed at $\mathrm{pH}$ 6. It is seen that the electrocatalytic response in acidic medium is higher than in basic medium. This indicates that the oxidation of AA involves the exchange of proton $[21,22]$ and hence is found to be stable in slightly acidic to neutral medium. Henceforth, for all the measurements, $\mathrm{pH} 6$ was chosen to be the optimum condition.

The effect of scan rates on the anodic peak currents for AA oxidation in $0.2 \mathrm{M}$ phosphate buffer solution on NiPc-NF-modified electrode in the presence of $5 \mathrm{mM}$ AA is studied. Figure 5a shows the cyclic voltammograms obtained in the potential range of -0.1 to $1.0 \mathrm{~V}$ for different scan rates from 0.1 to $0.3 \mathrm{~V} \mathrm{~s}^{-1}$. The anodic peak currents increase proportionately with increased scan rates. Figure $5 \mathrm{~b}$ shows
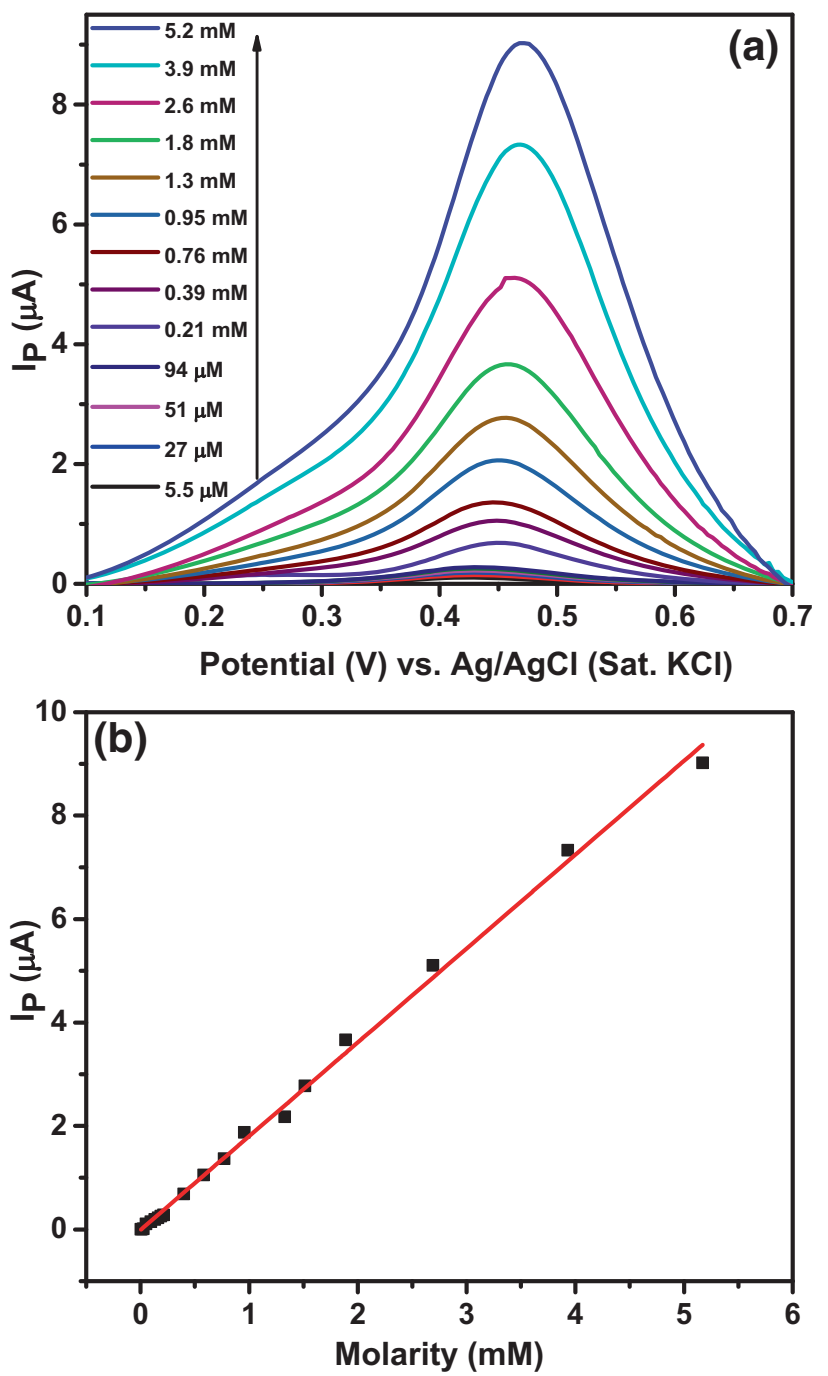

Figure 7. (a) Differential pulse voltammograms obtained for NiPc-NF-modified electrode at various concentrations from $5.5 \mu \mathrm{M}$ to $5.2 \mathrm{mM}$ ascorbic acid in $0.2 \mathrm{M}$ phosphate buffer solution of $\mathrm{pH}$ 6. (b) Calibration plot of anodic peak current vs. concentration of ascorbic acid for NiPc-NF-modified electrode.

that the plot of anodic peak current $\left(I_{\mathrm{P}}\right) v s$. square root of scan rate $\left(v^{1 / 2}\right)$ yields a straight line, which can be represented by a linear equation of the form $I_{\mathrm{P}}(\mu \mathrm{A})=$ $1.44 \pm 0.06\left(v^{1 / 2}\right)+0.11 \pm 0.02, R^{2}=0.986$. This indicates that the reactions occurring on the surface of the electrode are primarily diffusion-controlled processes.

Figure $5 \mathrm{c}$ shows the linear relationship between the anodic peak potential $\left(E_{\mathrm{P}}\right) v s . \log v$ and can be represented in the linear equation form, $E_{\mathrm{P}}(\mathrm{V})=0.060 \pm 0.003(\log v)+$ $0.322 \pm 0.007, R^{2}=0.974$. Number of electrons $(n)$ participating in the reaction can be calculated from the slope of the above plot $(\beta)$ using the Laviron equation [23] given as follows:

$$
\beta=\frac{2.303 R T}{\alpha n F},
$$




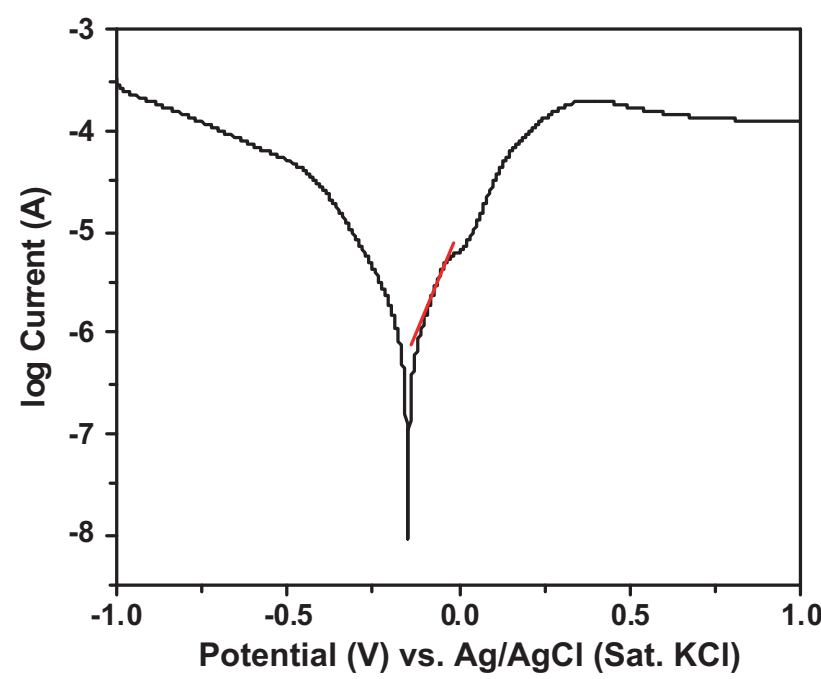

Figure 8. Tafel plot obtained for NiPc-NF-modified electrode in $0.2 \mathrm{M}$ phosphate buffer solution of $\mathrm{pH} 6$ with $5 \mathrm{mM}$ ascorbic acid.

where $\alpha$ is the charge transfer coefficient for irreversible or quasi-reversible reactions $(0.5), F$ the Faraday constant $\left(96,485.33 \mathrm{~s} \mathrm{~A} \mathrm{~mol}^{-1}\right), \quad R$ the ideal gas constant $\left(8.314 \mathrm{~J} \mathrm{~mol}^{-1} \mathrm{~K}^{-1}\right)$ and $T$ the absolute temperature $(300 \mathrm{~K})$. From this calculation, the value of $n$ is found to be 1.98 , indicating that the total number of electrons participating in AA oxidation is 2 [24].

The higher activity of the NiPc-NF-modified electrode in comparison with the commercial NiPc powder is clearly evident from figure 3 . Since the adsorption processes are dependent on the electrochemically active surface area, we have carried out $\mathrm{CV}$ measurements of the two electrodes in a standard $9 \mathrm{mM} \mathrm{K}_{3} \mathrm{FeCN}_{6}$ in phosphate solution of $\mathrm{pH} 6$ as shown in figure 6. The peaks appearing at 0.3 and $0.15 \mathrm{~V}$ belong to redox couple of ferricyanide ion represented in equation (2):

$$
\left[\mathrm{Fe}(\mathrm{CN})_{6}\right]^{3-}+\mathrm{e}^{-} \rightarrow\left[\mathrm{Fe}(\mathrm{CN})_{6}\right]^{4-}
$$

The electrochemically active surface area is calculated using the Randles-Sevcik formula given in equation (3) [16],

$$
I_{\mathrm{P}}=2.69 \times 10^{5} A D^{1 / 2} n^{3 / 2} \gamma^{1 / 2} C,
$$

where $I_{\mathrm{P}}$ is the peak current (A), $A$ the surface area of the electrode $\left(\mathrm{cm}^{2}\right), D$ the diffusion coefficient $(7.60 \times$ $\left.10^{-6} \mathrm{~cm}^{2} \mathrm{~s}^{-1}\right), n$ the number of electrons participating in the redox reaction $(n=1), \gamma$ the scan rate potential $\left(\mathrm{V} \mathrm{s}^{-1}\right)$ and $C$ the concentration of probe molecule in the bulk of the solution $\left(\mathrm{mol} \mathrm{cm} \mathrm{cm}^{-3}\right)$. The active surface area was found to be $3.61 \times 10^{-5}$ and $1.91 \times 10^{-5} \mathrm{~cm}^{2}$ for $\mathrm{NiPc}$ $\mathrm{NF}$ and commercial NiPc-modified electrodes, respectively.
The active surface area of the NiPc NF is almost twice that of commercial NiPc powder. This shows that the higher electrochemical activity of NiPc NF is due to its nanostructure morphology.

Electroanalytical determination of AA over varying concentration ranges is depicted in figure 7. Figure 7a shows the DPVs of NiPc-NF-modified electrodes over various concentrations of AA from 5.5 $\mu \mathrm{M}$ to $5.2 \mathrm{mM}$ in $0.2 \mathrm{M}$ phosphate buffer solution of $\mathrm{pH} 6$. The measurements were performed up to $25 \mathrm{mM}$ AA concentration; beyond this, the electrode response begins to deviate from linearity. The electroanalytical response of the modified electrode for AA sensing was found to be linear for a wide concentration range (from $5.5 \mu \mathrm{M}$ to $25 \mathrm{mM}$ ). The calibration plot for a lower concentration range, $5.5 \mu \mathrm{M}-5.2 \mathrm{mM}$ is shown in figure $7 \mathrm{~b}$ and the linearity equation can be represented as $I_{\mathrm{P}}(\mu \mathrm{A})=0.001$ (molarity) - $1.049 \times 10^{-8}, R^{2}=0.996$. The detection limit (LOD) for AA sensing was calculated as

$$
\mathrm{LOD}=\frac{3 \sigma}{m}
$$

where $\sigma$ is the standard deviation of the three blank DPV measurements and $m$ the slope obtained from the calibration curve. From this calculation, the LOD value was found to be $1.5 \mu \mathrm{M}$. These values are found to be on par with those of other MPc-composite-based electrodes for AA sensing. Detection limit from 0.3 to $2.1 \mu \mathrm{M}$ was achieved with nickel phthalocyanine immobilized on mesoporous $\mathrm{SiO}_{2}$-carbon matrices pre-treated with different catalysts [18], and $0.75 \mu \mathrm{M}$ for a substituted iron phthalocyanine [19]. Nano-sized cobalt phthalocyanine particles with carbon paste showed a detection limit of $1.7 \mu \mathrm{M}$ for AA in the presence of dopamine [20]. Zuo et al [21] demonstrated cobalt (II) phthalocyanine with multi-walled carbon nanotubes for AA oxidation with a detection limit of $1 \mu \mathrm{M}$.

Figure 8 shows the Tafel plot for NiPc-NF-modified electrode obtained at $\mathrm{pH} 6$ of $0.2 \mathrm{M}$ phosphate buffer solution in the presence of $5 \mathrm{mM}$ AA, which yields a Tafel slope of $118 \mathrm{mV} \mathrm{dec}^{-1}$. The number of electrons participating in the rate-determining step can be determined from equation (1) using the Tafel slope $(\beta)$. The value of $n$ is found to be 1.009 . Based on these calculations and according to the literature, the overall oxidation of AA is a two-electron process with two consecutive one electron transfers. This indicates that there is a loss of a single electron in AA oxidation to form a radical cation in the first step and then the subsequent loss of second electron to form dehydroascorbic acid in the second step $[21,24]$.

\section{Conclusions}

We have demonstrated the use of NiPc nanofibres prepared by a simple chemical route, as an electrochemical sensor for the detection of AA using an eco-friendly and cost-effective 
pencil graphite rod as the base electrode. The performance of the NiPc-NF-modified electrode is compared to that of the commercially available NiPc powder. The activity of NiPc $\mathrm{NF}$ is higher, which is ascribed to the higher electrochemically active surface area of the nanostructures. The electrode is quite stable despite not using any binding reagent or compositing with carbon materials to enhance its activity. However, it is anticipated that NiPc NF when combined with carbon materials would have higher sensitivity, lower oxidation potentials and stability towards electrochemical oxidation of a biomolecule in general. A linear calibration curve is achieved for the concentration range of AA from $5.5 \mu \mathrm{M}$ to $5.2 \mathrm{mM}$. The performance of the native NiPc NF for electrochemical oxidation of AA is on par with that of the reported MPccomposite-modified electrodes with a limit of detection of $1.5 \mu \mathrm{M}$.

\section{Acknowledgement}

We acknowledge financial assistance from DST FastTrack Project No. SR/FT/CS-170/2011.

\section{References}

[1] Madhuri K P, Kaur P, Ali M E and John N S 2017 J. Phys. Chem. C 1219249

[2] Vasudevan P, Phougat N and Shukla A K 1996 Appl. Organomet. Chem. 10591

[3] Chidembo A T, Ozoemena K I, Agboola B O, Gupta V, Wildgoose G G and Compton R G 2010 Energy Environ. Sci. 3 228

[4] Mani V, Devasenathipathy R, Chen S M, Gu J A and Huang S T 2015 Renew. Energy 74867
[5] Mori S, Nagata M, Nakahata Y, Yasuta K, Goto R, Kimura M et al 2010 J. Am. Chem. Soc. 1324054

[6] Yuan Y, Ahmed J and Kim S 2011 J. Power Sources 1961103

[7] Kottaichamy A R, Kotresh H M N, Devendrachari M C, Thimmappa R, Paswan B, Tiwari O et al 2015 J. Phys. Chem. C 11928276

[8] Wu H, Zeng M, Zhu X, Tian C, Mei B, Song Y et al 2018 ChemElectroChem 51

[9] Fadlallah M M, Eckern U, Romero A H and Schwingenschlogi U 2016 New J. Phys. 18013003

[10] Saito Y, Higuchi T, Sugimori H and Yabu H 2015 ChemNanoMat 192

[11] Mu J, Shao C, Guo Z, Zhang M, Zhang Z, Zhang P et al 2011 Nanoscale 35126

[12] Tong W Y, Djurisic A B, Xie M H, Ng A C M, Cheung K Y, Chan W K et al 2006 J. Phys. Chem. B 11017406

[13] Martin M G, Rodriguez-Mendez M L and de Saja J A 2010 Langmuir 2619217

[14] Li B, Kawakami T and Hiramatsu M 2003 Appl. Surf. Sci. 210 171

[15] Madhuri K P and John N S 2018 Appl. Surf. Sci. 449528

[16] Bramhaiah K, Pandey I, Singh V N, Kavitha C and John N S 2018 J. Nanopart. Res. 2056

[17] Wang X, Wu W, Ju H, Zou T, Qiao Z, Gong H et al 2016 Mater. Res. Express 3125002

[18] Barros S B A, Rahim A, Tanaka A A, Arenas L T, Landers R and Gushikem Y 2013 Electrochim. Acta 87140

[19] Oni J, Westbroek P and Nyokong T 2003 Electroanalysis 15 847

[20] Yang G J, Xu J J, Wang K and Chen Y 2006 Electroanalysis 18 282

[21] Zuo X, Zhang H and Li N 2012 Sens. Actuators B 1611074

[22] Ngai K S, Tan W T, Zainal Z, Zawawi R M and Zidan M 2013 Int. J. Electrochem. Sci. 810557

[23] Laviron E 1979 J. Electroanal. Chem. 10119

[24] Ruiz J J, Aldaz A and Dominguez M 1977 Can. J. Chem. 55 2799 